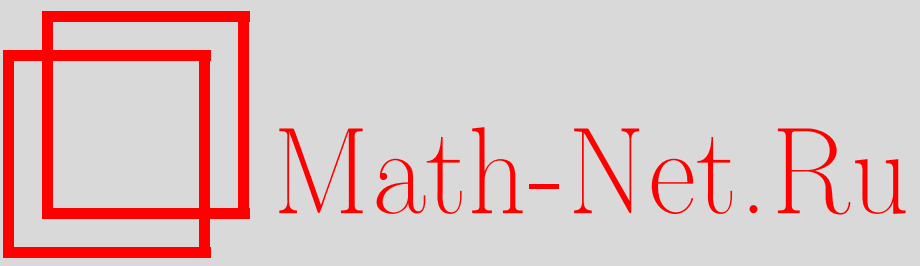

А. В. Костин, Н. Н. Костина, Задача Таммеса и контактное число сферы в пространствах постоянной кривизны, Итоги науки и техн. Сер. Соврем. мат. и ее прил. Темат. обз., 2020, том 182, 45-50

DOI: https://doi.org/10.36535/0233-6723-2020-182-45-50

Использование Общероссийского математического портала Math-Net.Ru подразумевает, что вы прочитали и согласны с пользовательским соглашением

http://www.mathnet.ru/rus/agreement

Параметры загрузки:

IP: 34.227 .88 .159

26 апреля 2023 г., $18: 16: 31$ 


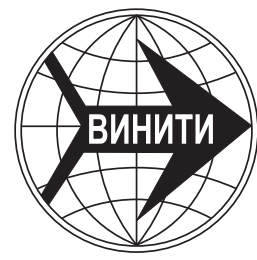

ИТОГИ НАУКИ И ТЕХНИКИ.

Современная математика и ее приложения.

Тематические обзоры.

Том 182 (2020). С. $45-50$

DOI: $10.36535 / 0233-6723-2020-182-45-50$

УДК 514.13, 514.17

\title{
ЗАДАЧА ТАММЕСА И КОНТАКТНОЕ ЧИСЛО СФЕРЫ В ПРОСТРАНСТВАХ ПОСТОЯННОЙ КРИВИЗНЫ
}

\author{
(c) 2020 г. $\quad$ А. В. КОСТИН, Н. Н. КОСТИНА
}

\begin{abstract}
АннотАция. В статье рассматривается задача о контактном числе сферы в трехмерном гиперболическом пространстве и трехмерном сферическом пространстве. Эта задача эквивалентна задаче Таммеса о нахождении максимально возможного значения минимумов сферических расстояний для множества из $N$ точек на единичной сфере евклидова пространства.
\end{abstract}

Ключевые слова: пространство постоянной кривизны, контактное число, задача Таммеса.

\section{TAMMES PROBLEM AND CONTACT NUMBER OF A SPHERE IN SPACES OF CONSTANT CURVATURE}

\author{
(c) 2020 A. V. KOSTIN, N. N. KOSTINA
}

\begin{abstract}
In this paper, we consider the problem on the contact number of a sphere in a threedimensional hyperbolic space and a three-dimensional spherical space.
\end{abstract}

Keywords and phrases: space of constant curvature, contact number, Tammes problem.

AMS Subject Classification: 53A35, 52B99

\section{1. Предварительные сведения.}

Определение 1. Контактным числом $k(n)$ в $n$-мерном пространстве называется наибольшее число непересекающихся шаров фиксированного радиуса, касающихся одного шара того же радиуса.

В евклидовом пространстве, вследствие наличия подобия, задачу нахождения контактного числа достаточно решить для шаров единичного радиуса. В двумерном случае ответ очевиден: $k(2)=6$. На двумерной сфере и на плоскости Лобачевского эта задача также решается элементарными средствами. В задачах подобного рода в пространствах ненулевой постоянной кривизны результат существенно зависит от линейных размеров фигур, в данном случае, от радиусов шаров (см., например, [2]). На плоскости Лобачевского контактное число не меньше шести и растет до бесконечности при увеличении радиуса кругов. Граничные значения, при которых меняется контактное число, легко определяются. На плоскости Лобачевского граничное значение радиуса равно половине стороны правильного треугольника с углами $2 \pi / n, n>6$. Число кругов, касающихся равного им круга на двумерной евклидовой сфере, не больше пяти и уменьшается с ростом радиуса кругов. Граничные значения этих радиусов также легко определяются. В трехмерном случае в евклидовом пространстве эту задачу можно сформулировать следующим образом: ККакое наибольшее число белых бильярдных шаров может одновременно касаться черного бильярдного шара?». Двенадцать белых шаров одинакового радиуса можно бесконечным числом способов расположить так, чтобы они касались центрального черного шара. Расположение двенадцати белых шаров в вершинах правильного икосаэдра обладает наибольшей симметрией. Но при этом 
шары можно сдвигать по поверхности так, что любые два шара можно поменять местами, не отрывая от черного шара. Элементарными геометрическими соображениями определяется завышенная верхняя оценка контактного числа: отношение площади двумерной евклидовой сферы к площади проекции на нее касающегося шара того же радиуса равно

$$
\operatorname{cosec}^{2} \frac{\pi}{12} \approx 14,9282 \ldots
$$

Такая оценка стала причиной предположения, что, сдвинув белые шары, между ними можно вставить еще один. Вопрос о контактном числе обсуждался Исааком Ньютоном и Дэвидом Грегори в 1694 году. Грегори допускал, что контактное число равно 13, Ньютон же считал, что оно равно 12. Нижняя оценка (12) была известна еще с античных времен. Задачу о контактном числе в трехмерном пространстве называют проблемой тринадцати шаров или проблемой НьютонаГрегори. Только в 1953 году К. Шютте и Б. Л. ван дер Варден доказали (см. [25]), что контактное число в трехмерном евклидовом пространстве равно 12. В настоящее время существует много различных доказательств этого утверждения, часть из которых приведена в [5, 8, 15-17]. Проблема 13 шаров допускает следующее обобщение, впервые сформулированное ботаником Таммесом в работе [26] «О происхождении числа и расположения мест выхода на поверхность пыльцевых зерен»: какой вид имеет множество из $N$ точек на двумерной сфере евклидова пространства, если наименьшее расстояние между точками этого множества максимально возможное? Л. Фейеш Тот решил данную задачу в 1943 г. для $N=3,4,6,12$ (см. [13, 14]), К. Шютте и Б. ван дер Варден в 1951 г. — - для $N=5,7,8,9$ (см. [24]), Л. Данцер в 1963 г. 一 для $N=10,11$ (см. [7, 11, 12]), Р. М. Робинсон в 1961 г. - для $N=24$ (см. [23]). Дальнейшие продвижения в этом направлении - нахождение максимально возможного значения минимумов сферических расстояний для $N=13$ и $N=14$ - осуществлены О. Р. Мусиным и А. С. Тарасовым (см. [9, 19, 20]). Контактные числа в евклидовом пространстве кроме размерности 3 (и тривиальных случаев в размерностях 1 и 2) найдены в размерностях 4 (О. Р. Мусин), 8, 24 (В. И. Левенштейн и независимо А. Олдыжко и Н. Слоэн; см. $[4,10,18,21,22])$. Рассматриваемая задача о контактном числе сферы имеет множество приложений в различных областях науки и техники, в частности, в теории передачи информации. Она представляет собой частный случай задачи о сферическом коде (см. [1]).

2. Интерпретация задачи Таммеса. Привлечение неевклидовых геометрий при рассмотрении вопросов, касающихся евклидовых пространств, позволяет эти вопросы по-иному интерпретировать (см., например, [3]). На задачу Таммеса также можно взглянуть с другой точки зрения. Верхняя грань $d_{N}$ наименьшего из расстояний между $N$ точками на двумерной евклидовой сфере единичного радиуса определяет предельное значение радиуса шара в трехмерном пространстве постоянной кривизны, которого касаются $N$ равных ему шаров. Если значение $d_{N}<\pi / 3$, то оно определяет предельное значение радиуса шара в пространстве Лобачевского, если $d_{N}>\pi / 3$, то им определяется соответствующее значение радиуса шара в сферическом пространстве.

3. Контактное число сферы в гиперболическом пространстве. Контактное число сферы в гиперболическом пространстве зависит от радиуса сферы. Во всех размерностях оно не меньше, чем контактное число сферы в евклидовом пространстве, и с увеличением радиуса сферы растет до бесконечности. Для оценки этого числа можно использовать оценки для максимальных значений минимумов сферических расстояний между $N$ точками на единичной сфере евклидова пространства. Пусть $x, y$ - точки на сфере $S^{2}$ единичного радиуса в трехмерном евклидовом пространстве. Обозначим через $d(x, y)$ расстояние между точками $x$ и $y$ во внутренней геометрии сферы. Это расстояние совпадает с центральным углом, определяемым точками $x$ и $y$. Пусть $M$ - множество, состоящее из $N$ точек на единичной сфере $S^{2}$. Обозначим при несовпадающих $x$ и $y$ из $M$ через $\phi(M)$ наименьшее расстояние между точками этого множества, т.е.

$$
\phi(M)=\min _{(x, y \in M)} d(x, y)
$$

а наибольшее значение, которое может достигать $\phi(M)$ для множества из $N$ точек на единичной сфере, через $d_{N}$. Тогда при $N \geqslant 13$ имеет место следующее утверждение. 
Теорема 1. Контактное число сферы радиуса $R$ в трехмерном гиперболическом пространстве $H^{3}$ кривизны $K=-1 / \sigma^{2}$ равно $N(N \geqslant 13)$, если радиус сферы удовлетворяет условию

$$
\frac{\sigma}{2} \cdot \operatorname{arch} \frac{\cos \left(d_{N}\right)}{1-\cos \left(d_{N}\right)} \leqslant R<\frac{\sigma}{2} \cdot \operatorname{arch} \frac{\cos \left(d_{N+1}\right)}{1-\cos \left(d_{N+1}\right)}
$$

$u d_{N} \neq d_{N+1}$. Если при каком-либо значении $N$ имеет место равенство $d_{N}=d_{N+1}$, то контактное число сферы в гиперболическом пространстве не может принимать значение $N$ ни для каких радиусов сферы.

Доказательство. Пусть $O$ - центр черного шара, $X$ и $Y$ - центры белых шаров радиуса $R$ в пространстве Лобачевского, расстояние между точками касания которых с черным шаром минимально. Тогда в треугольнике $O X Y$ имеем: $\angle X O Y=d_{N}$. Граничное значение радиуса $R$ определяется условием, когда все три шара касаются друг друга. Центры их образуют правильный треугольник со стороной $2 R$. В двойственную теорему косинусов гиперболической геометрии

$$
\cos C=-\cos A \cdot \cos B+\sin A \cdot \sin B \cdot \operatorname{ch}\left(\frac{c}{\sigma}\right)
$$

подставим $A=B=C=d_{N}, c=2 R$ :

$$
\cos \left(d_{N}\right)=-\cos ^{2}\left(d_{N}\right)+\sin ^{2}\left(d_{N}\right) \cdot \operatorname{ch}\left(\frac{2 R}{\sigma}\right)
$$

Из (2) получим:

$$
\operatorname{ch}\left(\frac{2 R}{\sigma}\right)=\frac{\cos \left(d_{N}\right)}{1-\cos \left(d_{N}\right)}
$$

Если $R_{N}\left(R_{N+1}\right)$ - минимальный радиус черного шара, которого касаются $N$ (соответственно $N+1)$ непересекающихся белых шаров в пространстве Лобачевского, то

$$
R_{N}=\frac{\sigma}{2} \cdot \operatorname{arch} \frac{\cos \left(d_{N}\right)}{1-\cos \left(d_{N}\right)}, \quad R_{N+1}=\frac{\sigma}{2} \cdot \operatorname{arch} \frac{\cos \left(d_{N+1}\right)}{1-\cos \left(d_{N+1}\right)} .
$$

Отсюда следует первая часть утверждения теоремы (соотношение (1)). Вторая часть утверждения очевидна.

Следствие 1. Контактное число сферы $S_{h}^{2}$ в трехмерном гиперболическом пространстве совпадает с контактным числом сферы $S^{2}$ евклидова пространства тогда и только тогда, когда радиус сферы $S_{h}^{2}$ меньше, чем

$$
\frac{\sigma}{2} \cdot \operatorname{arch} \frac{\cos \left(d_{13}\right)}{1-\cos \left(d_{13}\right)} \approx \frac{\sigma}{2} \cdot 0,3000768 \ldots
$$

Значения $d_{13} \approx 57,1367^{\circ}$ и $d_{14} \approx 55,67057^{\circ}$, как уже было сказано выше, найдены О. Р. Мусиным и А. С. Тарасовым (см. $[19,20])$. Наименьший радиус сферы в пространстве Лобачевского, контактное число которой равно 14 , приближенно равен $R_{14} \approx(\sigma / 2) \cdot 0,37416789 \ldots$.

4. Оценки связей контактного числа сферы в гиперболическом пространстве с ее радиусом. Первоначально оценим сверху контактное число сферы радиуса $R$ в трехмерном пространстве Лобачевского.

Лемма 1. Контактное число $k_{R}\left(H^{3}\right)$ сферы радиуса $R$ в пространстве Лобачевского кривизни $K=-1 / \sigma^{2}$ удовлетворяет условию

$$
k_{R}\left(H^{3}\right)<\operatorname{cosec}^{2}\left(\frac{1}{4} \cdot \arccos \frac{\operatorname{ch}\left(\frac{2 R}{\sigma}\right)}{1+\operatorname{ch}\left(\frac{2 R}{\sigma}\right)}\right) .
$$

Доказательство. Площадь двумерной сферы $S_{h}^{2}$ радиуса $R$ в трехмерном пространстве Лобачевского кривизны $K=-1 / \sigma^{2}$ равна

$$
S_{h}^{2}=4 \pi \sigma^{2} \operatorname{sh}^{2}\left(\frac{R}{\sigma}\right) .
$$


Спроектируем из центра этой сферы на ее поверхность касающийся шар того же радиуса. Проекцией шара будет круг радиуса

$$
r=\frac{\sigma}{2} \cdot \operatorname{sh}\left(\frac{R}{\sigma}\right) \cdot \arccos \frac{\operatorname{ch}\left(\frac{2 R}{\sigma}\right)}{1+\operatorname{ch}\left(\frac{2 R}{\sigma}\right)} .
$$

Поскольку на сфере радиуса $R$ в трехмерном пространстве Лобачевского кривизны $K=-1 / \sigma^{2}$ реализуется геометрия евклидовой сферы радиуса $\sigma \operatorname{sh}(R / \sigma)$, площадь круга, являющегося проекцией шара, будет равна

$$
S_{c}=4 \pi \sigma^{2} \operatorname{sh}^{2}\left(\frac{R}{\sigma}\right) \cdot \sin ^{2}\left(\frac{r}{\sigma \operatorname{sh}\left(\frac{R}{\sigma}\right)}\right)
$$

(площадь круга радиуса $r$ на евклидовой сфере радиуса $\rho$ равна $4 \pi \rho^{2} \sin ^{2}(r /(2 \rho))$ ). Подставив значение $r$ из (6) в (7) и поделив затем (5) на полученное выражение, получим оценку (4):

$$
k_{R}\left(H^{3}\right)<\frac{S_{h}^{2}}{S_{c}}=\operatorname{cosec}^{2}\left(\frac{1}{4} \cdot \arccos \frac{\operatorname{ch}\left(\frac{2 R}{\sigma}\right)}{1+\operatorname{ch}\left(\frac{2 R}{\sigma}\right)}\right) .
$$

Замечание 1. Если в (4) радиус сферы $R$ будет мал по сравнению с $\sigma$, то правая часть неравенства перейдет в

$$
\operatorname{cosec}^{2}\left(\frac{1}{4} \cdot \arccos \frac{\operatorname{ch}\left(\frac{2 R}{\sigma}\right)}{1+\operatorname{ch}\left(\frac{2 R}{\sigma}\right)}\right) \rightarrow \operatorname{cosec}^{2}\left(\frac{\pi}{12}\right) \approx 14,9282 \ldots,
$$

т.е. в соответствующую евклидову оценку.

Приведем теперь оценку снизу для радиуса сферы в гиперболическом пространстве в зависимости от контактного числа. Л. Фейеш Тот нашел следующую оценку для максимальных значений минимумов сферических расстояний для $N$ точек на сфере единичного радиуса в евклидовом пространстве:

$$
d_{N} \leqslant \arccos \frac{C_{N}}{1-C_{N}}, \quad C_{N}=\cos \left(\frac{\pi \cdot N}{3 N-6}\right) .
$$

Используя эту оценку, получим следующее утверждение.

Лемма 2. Если контактное число сферы в гиперболическом пространстве равно $N$, то ее радиус не меньше, чем

$$
\frac{\sigma}{2} \cdot \operatorname{arch} \frac{\cos \left(\frac{\pi \cdot N}{3 N-6}\right)}{1-\cos \left(\frac{\pi \cdot N}{3 N-6}\right)}
$$

Доказательство. При уменьшении величины угла правильного треугольника в гиперболическом пространстве длина его стороны увеличивается. С учетом этого, подставив в (3) оценки Фейеша Тота, получим нижнюю оценку (9) для радиуса сферы в гиперболическом пространстве.

5. Контактное число сферы в сферическом пространстве. Контактное число сферы в сферическом пространстве любой размерности не больше, чем контактное число в евклидовом пространстве той же размерности. Значения $d_{N}$ при $N<13$, найденные Фейешем Тотом, Шютте, ван дер Варденом и Данцером для сферы трехмерного евклидова пространства, дают полное решение задачи в трехмерном сферическом пространстве (для $N<3$ решение тривиально). Из этих результатов, в частности, следует, что контактное число сферы в этом пространстве не может равняться пяти и одиннадцати, так как значения $d_{5}$ и $d_{6}$ (соответственно $d_{11}$ и $d_{12}$ ) равны между собой. Оценки (8) Фейеша Тота для $d_{6}$ и $d_{12}$ являются точными. В отличие от гиперболического 
случая, контактное число будет равно $N \leqslant 12$, если $R_{N+1}<R \leqslant R_{N}$ и $d_{N} \neq d_{N+1}$, где граничные значения радиусов определяются из правильного сферического треугольника с углами $d_{N}$. Отметим также, что поскольку

имеет место утверждение.

$$
\cos \left(d_{12}\right)=\frac{1}{\sqrt{5}}<\frac{1}{2}
$$

Лемма 3. Контактное число двумерной сферы в трехмерном сферическом пространстве кривизны $K=1 / \rho^{2}$ совпадает с евклидовым тогда и только тогда, когда радиус сферы не превосходит значения

$$
\frac{\rho}{2} \cdot \arccos \frac{\sqrt{5}+1}{4} \approx \rho \cdot 0,314159265 \ldots
$$

Доказательство. Максимум минимальных расстояний между двенадцатью точками на сфере достигается для конфигурации точек в вершинах правильного икосаэдра. Отсюда получаем указанное выше значение $\cos \left(d_{12}\right)=\frac{1}{\sqrt{5}}$. Применив для правильного сферического треугольника со сторонами $2 R$ и углами $d_{12}$ двойственную теорему косинусов сферической геометрии:

$$
\cos C=-\cos A \cdot \cos B+\sin A \cdot \sin B \cdot \cos \frac{c}{\rho},
$$

где $A=B=C=d_{12}, c=2 R$, получим предельное значение радиуса сферы в трехмерном сферическом пространстве, контактное число которой равно двенадцати.

6. Заключительные замечания. Используя результаты работ $[6,18,21]$, можно получить другие оценки контактных чисел в пространствах постоянной кривизны в различных размерностях. Кроме того, в пространстве Лобачевского, наряду с числом «целующих» сфер («kissing number» - терминология, закрепившаяся в англоязычной литературе, отсюда, в частности, обозначение $k(N)$ ), в вопросах выпуклого анализа представляет интерес определение числа «целующих» плоскостей, «целующих» орисфер для сфер различных радиусов.

\section{СПИСОК ЛИТЕРАТУРЫ}

1. Зиновъев В. А., Эриксон Т. Новые нижние оценки на контактное число для небольших размерностей// Пробл. передачи информ. - 1999. - 35, № 4. - С. 3-11.

2. Костин А. В. Задача о тени в пространстве Лобачевского// Укр. мат. ж. - 2018. - 70, № 11. C. $1525-1532$.

3. Костин А. В., Костина Н. Н. Интерпретации теоремы Кези и ее гиперболического аналога// Сиб. электрон. мат. изв. $-2016 .-13 .-$ С. 242-251.

4. Левенштейн В. И. О границах для упаковок в $n$-мерном евклидовом пространстве// Докл. АН СССР. - 1979. - 245, № 6. - С. 1299-1303.

5. Anstreicher K. The thirteen spheres: A new proof// Discr. Comput. Geom. - 2004. - 31. - P. 613-625.

6. Bachoc C., Vallentin F. New upper bounds for kissing numbers from semidefinite programming// Discr. Comput. Geom. - 2008. - 21. - P. 909-924.

7. Böröczky K. The problem of Tammes for $n=11 / /$ Stud. Sci. Math. Hungar. - 1983. - 18. - P. 165-171.

8. Böröczky K. The Newton-Gregory problem revisited// in: Discrete Geometry (Bezdek A., ed.). - Marcel Dekker, 2003. - P. 103-110.

9. Böröczky K., Szabó L. Arrangements of 13 points on a sphere// in: Discrete Geometry (Bezdek A., ed.). Marcel Dekker, 2003. - P. 111-184.

10. Boyvalenkov P., Dodunekov S, Musin O. R. A survey on the kissing number// Serd. Math. J. — 2012. 38. - P. 507-522.

11. Danzer L. Endliche Punktmengen auf der 2-Sphäre mit möglichst grossen Minimalabstand. - Universität Göttingen, 1963.

12. Danzer L. Finite point-sets on $\mathbb{S}^{2}$ with minimum distance as large as possible// Discr. Math. — 1986. 60. - P. 3-66.

13. Fejes Tóth L. Über die Abschätzung des kürzesten Abstandes zweier Punkte eines auf einer Kugelfäche liegenden Punktsystems// Jber. Deutsch. Math. Verein. - 1943. - 53. - P. 66-68.

14. Fejes Tóth L. Lagerungen in der Ebene, auf der Kugel und in Raum. - Berlin: Springer-Verlag, 1953. 
15. Maehara H. Isoperimetric theorem for spherical polygons and the problem of 13 spheres// Ryukyu Math. J. - 2001. - 14. - P. 1770-1778.

16. Maehara H. The problem of thirteen spheres - a proof for undergraduates// Eur. J. Combin. — 2007. 28. - P. 41-57.

17. Musin O. R. The kissing problem in three dimensions// Discr. Comput. Geom. - 2006. - 35. - P. 375-384.

18. Musin O. R. The kissing problem in four dimensions// Ann. Math. - 2008. - 168. - P. 1-32.

19. Musin O. R., Tarasov A. C. The strong thirteen spheres problem// Discr. Comput. Geom. - 2012. - 48. - P. 128-141.

20. Musin O. R., Tarasov A. C. The Tammes problem for $N=14 /$ arXiv: 1410.2536 [math.MG] .

21. Odlyzko A. M., Sloane N. J. A. New bounds on the number of unit spheres that can touch a unit sphere in $n$ dimensions// J. Combin. Th. A. - 1979. - 26. - P. 210-214.

22. Pfender F., Ziegler G. M. Kissing numbers, sphere packings, and some unexpected proofs// Not. Am. Math. Soc. $-2004 .-51$. - P. 873-883.

23. Robinson R. M. Arrangement of 24 circles on a sphere// Math. Ann. - 1961. - 144. - P. 14-48.

24. Schütte K., Waerden, van der B. L. Auf welcher Kugel haben 5, 6, 7,8 oder 9 Punkte mit Mindestabstand 1 Platz?// Math. Ann. - 1951. - 123. - P. 96-124.

25. Schütte K., van der Waerden B. L. Das Problem der dreizehn Kugeln// Math. Ann. — 1953. - 125. P. 325-334.

26. Tammes R. M. L. On the origin number and arrangement of the places of exits on the surface of pollengrains// Rec. Trv. Bot. Neerl. - 1930. - 27. - P. 1-84.

Костин Андрей Викторович

Казанский (Приволжский) федеральный университет, Елабужский институт, Елабуга

E-mail: kostin_andrei@mail.ru

Костина Наталья Николаевна

Казанский (Приволжский) федеральный университет, Елабужский институт, Елабуга

E-mail: natnikost@mail.ru 